\title{
A Method of the UMTS-FDD Network Design Based on Universal Load Characteristics
}

\author{
Slawomir Gajewski \\ Gdansk University of Technology, \\ 11/12 Narutowicza Str., PL-80-233 Gdansk, Poland \\ slagaj@eti.pg.gda.pl
}

\begin{abstract}
In the paper an original method of the UMTS radio network design was presented. The method is based on simple way of capacity-coverage tradeoff estimation for WCDMA/FDD radio interface. This trade-off is estimated by using universal load characteristics and normalized coverage characteristics. The characteristics are useful for any propagation environment as well as for any service performance requirements. The practical applications of these characteristics on radio network planning and maintenance were described.
\end{abstract}

\section{Introduction}

The WCDMA/FDD radio interface provides various ways of its resources utilization for UMTS radio network planning [1,2,3]. The most effective utilization of these resources depends however, on the knowledge of relationships among all the techniques used in that interface and their impact on the transmission performance, system capacity and coverage in real conditions. The coverage of mobile and base stations depends in turn on interface load that has influence on system capacity [4]. It is well known that system capacity is dependent on transmission performance and traffic characteristics required for different services. Capacity also depends on propagation environment and speed of terminals [3,5]. In addition, all these characteristics change over time.

Methods for estimating a coverage using characteristics defining the maximum allowable pathloss in the radio channel, allowing the performance of services are well known. A novel approach described in this paper relies on the use of the family of load characteristics to determine the capacity-coverage trade-off. It permits the quantitative assessment of benefits and losses arising from the use of this phenomenon during the design and operation of the UMTS networks.

The capacity-coverage trade-off estimation is facilitated by the designation of a single parameter of the system. This parameter can be easily calculated on the basis of link budget or can be estimated using the method described in [6].

\section{WCDMA/FDD Radio Interface Load}

The load of WCDMA/FDD radio interface in a given cell depends on multiple access interference coming from that cell and surrounding cells. As known, excessively high 
value of load cannot be accepted in the system. Coverage of the mobile and base stations can be reduced significantly for heavy-load and many services may be inaccessible. Accordingly, when calculating the link budget we have to take into account the interference margin (usually $3 \mathrm{~dB}$ ) [2].

In the case of uplink transmission the load factor $\eta_{U L}$ can be found $[2,3]$ according to the following expression

$$
\eta_{U L}=\left(1+\bar{\xi}_{U L}\right) \sum_{j=1}^{M} \frac{v_{j}}{d_{p c, j}} \frac{\left(E_{b} / N_{t}\right)_{n, j}}{G_{j}}
$$

where $M$ is the number of connections at a given time, $v_{j}$ is the activity factor of signal source (for $j$-th connection), $d_{p c, j}$ is the factor of dynamic power control efficiency for $j$-th connection, $\bar{\xi}_{U L}$ is the average value of other-cell to own-cell interference ratio in uplink, $G_{j}$ is the processing gain for $j$-th connection, $\left(E_{b} / N_{t}\right)_{n, j}$ is the required (nominal) bit energy per interference and noise power density ratio for $j$-th connection (for the service implemented in this connection).

Maximum allowable pathloss $L_{\max , U L, j}$ for the uplink $j$-th connection is related to the load factor $\eta_{U L}$ by

$$
L_{\max , U L, j}=\frac{P_{M S, j} G_{T x} G_{R x} G_{S H O}}{F_{T x} F_{R x} N} \frac{G_{j}}{\left(E_{b} / N_{t}\right)_{n, j}}\left(1-\eta_{U L}\right)
$$

where $P_{M S, j}$ is the power of signals transmitted by the mobile station for $j$-th connection, $G_{T x}$ is the transmitter antenna gain, $G_{R x}$ is the receiver antenna gain, $F_{T x}$ is the signal loss of transmitter antenna feeder, $F_{R x}$ is the signal loss of receiver antenna feeder, $G_{S H O}$ is a soft handover gain, $N$ is the power of thermal noise (including the receiver noise figure).

Expression (2) is called the uplink load characteristic [3] of $j$-th connection. This characteristic can be used to determine the range of a mobile stations.

Additionally, the interface load $\eta_{D L}$ for downlink can be defined on similar principle as for uplink including average value of downlink orthogonality factor $\bar{\alpha}$ [3]. It is given by

$$
\eta_{D L}=\left(1-\bar{\alpha}+\bar{\xi}_{D L}\right) \sum_{j=1}^{M} \frac{v_{j}}{d_{p c, j}} \frac{\left(E_{b} / N_{t}\right)_{n, j}}{G_{j}}
$$

where $\bar{\alpha}$ represents the average orthogonality factor, $\bar{\xi}_{D L}$ is the average value of other-cell to own-cell interference ratio in downlink.

In this case the maximum allowable pathloss $L_{\max , D L, j}$ for $j$-th connection is related to the load factor $\eta_{D L}$ by the following expression

$$
L_{\max , D L, j}=\frac{P_{B S, j} G_{T x} G_{R x} G_{S H O}}{F_{T x} F_{R x} N} \frac{G_{j}}{\left(E_{b} / N_{t}\right)_{n, j}}\left(1-\eta_{D L}\right)
$$


when $P_{B S, j}$ is the power of signals transmitted by the base station over the traffic channel in $j$-th connection [3,6,7]. Expression (4) is called downlink load characteristic of $j$-th type of service.

It was proved in $[3,6]$, that the maximum allowable pathloss $L_{\max , D L}$ for all connections (and all types of implemented services) not always depend on the type of service and it can be calculated from expression

$$
L_{\max , D L}=\frac{P_{B S, \max }}{N} \frac{G_{T x} G_{R x} G_{S H O}}{F_{T x} F_{R x}}\left(1-\bar{\alpha}+\bar{\xi}_{D L}\right)\left(\frac{1}{\eta_{D L}}-1\right)
$$

when $P_{B S \text {, max }}$ is the maximum power of signals transmitted by the base station to all active mobile stations.

The expression (5) is called the generalized downlink load characteristic [3] of the WCDMA/FDD radio interface. This characteristic is valid for different services having various performance requirements and data rates with the limitations characterized in [3]. Expression (5) can be used to estimate the coverage and capacity-coverage trade-off for mixed services of any type.

\section{Family of Universal Load Characteristics in Uplink}

As we can see in expression (2), maximum allowable uplink pathloss $L_{\max , U L, j}$ for the $j$-th connection can be calculated on the basis of link budget. We can introduce the parameter $A_{U L, j}$ resulting from uplink link budget, in form

$$
A_{U L, j}=\frac{P_{M S, j} G_{T x} G_{R x} G_{S H O} G_{j}}{F_{T x} F_{R x}\left(E_{b} / N_{t}\right)_{n, j} N}
$$

Then we can describe the load characteristic, which is simplified to the form

$$
L_{\max , U L, j}=A_{U L, j}\left(1-\eta_{U L}\right)
$$

Now, the relationship between $L_{m a x U L, j}$ and $\eta_{U L}$ depends only on single parameter $A_{U L, j}$. On the other hand, the $A_{U L, j}$ parameter of the uplink load characteristic depends on the mobile station transmitter power and another radio link budget parameters. The power of transmitted signals can change and the value of the parameter $A_{U L, j}$ can also be changeable in the time.

If we have a realistic estimate of possible values for the parameter $A_{U L, j}$ then we can draw the family of uplink load characteristics. The family reflects the relationship between $L_{\max , U L, j}$ and $\eta_{U L}$ for different parameters of radio link budget. The family is shown in Fig. 1.

In this simple case, through the use of the load characteristics we can determine the scope of variation of maximum allowable pathloss $\left(L_{\max , U L, j}\right)$ for any services and for a specific load. Also, we can determine the pathloss variability when changing load, or we can estimate the extent of the coverage variation.

As we can see, the family of load characteristics can be used to cellular network design, as explained in section 6 and 7. 


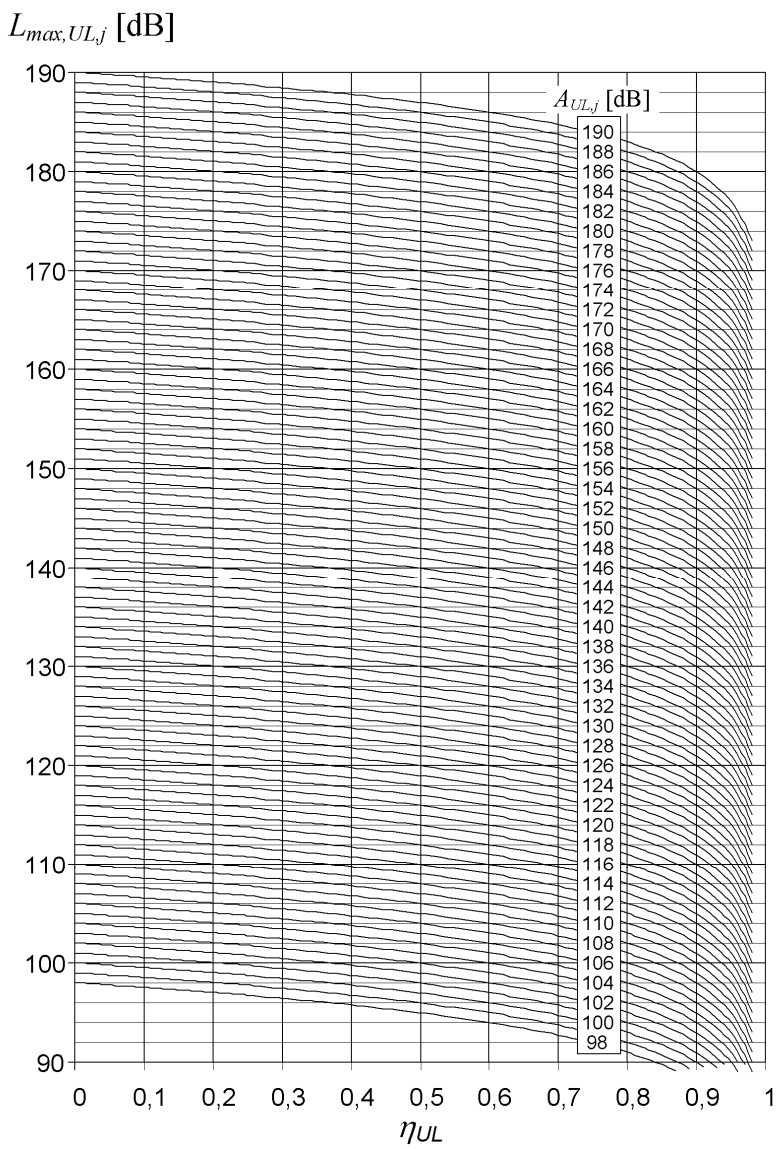

Fig. 1. Family of load characteristics for uplink designed using the link budget

\section{Families of Universal Load Characteristics in Downlink}

In downlink, maximum allowable pathloss $L_{m a x, D L, j}$ for $j$-th connection, we can calculate from expression (4) (on the basis of link budget) and then we have

$$
L_{\max , D L, j}=A_{D L, j}\left(1-\eta_{D L}\right)
$$

where parameter $A_{D L, j}$ has the form

$$
A_{D L, j}=\frac{P_{B S, j} G_{T x} G_{R x} G_{S H O} G_{j}}{F_{T x} F_{R x}\left(E_{b} / N_{t}\right)_{n, j} N}
$$




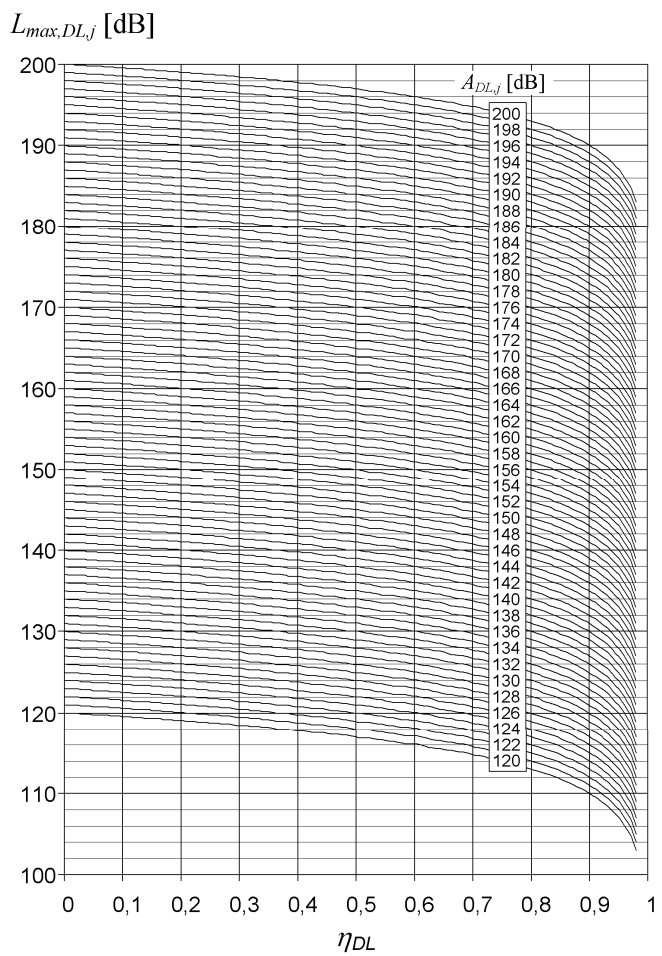

Fig. 2. Family of load characteristics for downlink designed using the link budget

If we have a realistic estimate of possible values for the parameter $A_{D L, j}$ then we can draw the family of downlink load characteristics, as we can see in Fig. 2.

We can also use another method for calculation of the downlink load characteristics, which is presented by details in [6]. Then the family of downlink load characteristics we can calculate using formula (5).

Then the maximum allowable pathloss $L_{\max , D L}[3,6]$ for the service of any type (implemented in downlink connection) is given by

$$
L_{\max , D L}=A_{D L}\left(1 / \eta_{D L}-1\right)
$$

where

$$
A_{D L}=\frac{P_{B S, \max }\left(1-\bar{\alpha}+\bar{\xi}_{D L}\right) G_{T x} G_{R x} G_{S H O}}{F_{T x} F_{R x} N}
$$

The graph of the family of downlink load characteristics calculated using the expression (5) is presented in Fig. 3. 


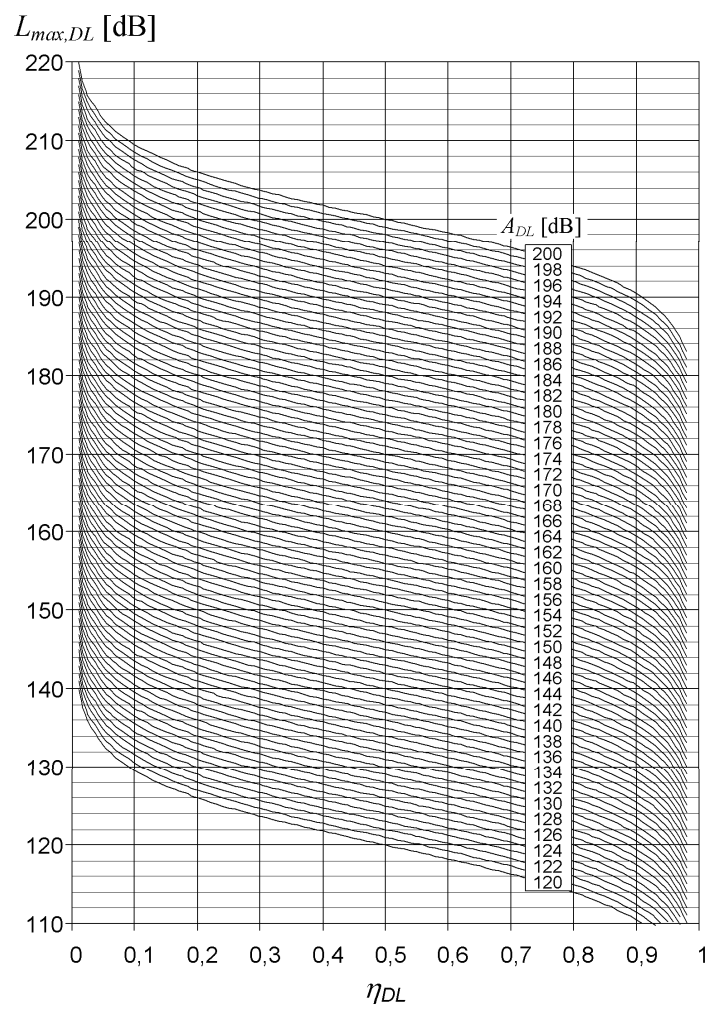

Fig. 3. Family of downlink load characteristics designed using the formula (5)

\section{Normalized Coverage Characteristics}

In order to evaluate the coverage on the basis of universal load characteristics, the propagation loss characteristic is needed. For that purpose, we can use any empirical propagation loss model. For example, we can use standardized ITU-R propagation models published in [8].

In particular for microcell in UMTS i.e. for the Outdoor to Indoor \& Pedestrian (OIP) propagation environment the propagation loss $L_{\text {prop }}$ can be estimated as

$$
L_{\text {prop }}[d B]=148+40 \lg (d)
$$

where the range $d$ is given in [km].

For example, in uplink we can calculate the range of mobile station using the expression (2) for the service implemented in $j$-th connection. If we compare the maximum allowable pathloss with the propagation loss of a given microcell propagation environment $L_{\text {max }, U L, j}=L_{\text {prop }}$ then we get

$$
A_{U L, j}[d B]+10 \lg \left(1-\eta_{U L}\right)=148[d B]+40 \lg \left(d_{U L, j}\right)
$$

where $d_{U L, j}$ is the range of mobile station for the service implemented in $j$-th connection. 
The range of mobile station is given by

$$
d_{U L, j}=10^{\frac{D_{U L, j}[d B]+10 \lg \left(1-\eta_{U L}\right)}{40}}
$$

where parameter $D_{U L, j}$ has the form

$$
D_{U L, j}[d B]=A_{U L, j}[d B]-148[d B]
$$

Parameter $D_{U L, j}$ is the value of $A_{U L, j}$ normalized by the constant propagation loss in a given propagation environment, i.e. normalized by $148 \mathrm{~dB}$ as in our case.

Also, for the case of downlink transmission and the same propagation loss model we can calculate the base station range (for $j$-th connection) using load characteristic given by (4). Then we have

$$
A_{D L, j}[d B]+10 \lg \left(1-\eta_{D L}\right)=148[d B]+40 \lg \left(d_{D L, j}\right)
$$

The base station range $d_{D L, j}$ for the service implemented in $j$-th connection can be calculated from

$$
d_{D L, j}=10 \frac{D_{D L, j}[d B]+10 \lg \left(1-\eta_{D L}\right)}{40}
$$

Parameter $D_{D L, j}$ is now given by expression

$$
D_{D L, j}[d B]=A_{D L, j}[d B]-148[d B]
$$

On the other hand, the base station range $d_{D L}$ for the service of any type and for the mixed set of implemented services can be estimated using universal load characteristic given by the formula (5). Then we get

$$
A_{D L}[d B]+10 \lg \left(1 / \eta_{D L}-1\right)=148[d B]+40 \lg \left(d_{D L}\right)
$$

In this case, the base station range $d_{D L}$ for the service of any type has the form

$$
d_{D L}=10 \frac{\frac{D_{D L}[d B]+10 \lg \left(1 / \eta_{D L}-1\right)}{40}}{40}
$$

Then the parameter $D_{D L}$ is given by

$$
D_{D L}[d B]=A_{D L}[d B]-148[d B]
$$

Using expressions (14), (17) and (20) we can draw the families of normalized coverage characteristics for a given propagation model for both the uplink and downlink transmission. Sample graph of the family of normalized coverage characteristics for uplink is presented in Fig. 4 (shown in section 6).

\section{Example Network Design Procedures}

For example, let $D_{U L, j}=9 \mathrm{~dB}$ and the value of uplink load $\eta_{1, U L}=0.5$ for a given connection $j$ provided the same service for all connections is used. Then the mobile 
station range $d_{U L, j}$ is approximately $1.3 \mathrm{~km}$, as we can see on the relevant coverage characteristic in Fig. 6.1.

For the cell radius decreased from $1.3 \mathrm{~km}$ to $1 \mathrm{~km}$ the value of $D_{U L, j}$ is decreased to $6 \mathrm{~dB}$. We may obtain the same result by increasing the load factor to $\eta_{U L, 2} \approx 0.87$ as is shown in Fig. 4. It gives the capacity growth $\Delta M$ in proportion to the ratio of discussed load factors i.e.

$$
\Delta M=\frac{\eta_{U L, 2}}{\eta_{U L, 1}}=\frac{0.87}{0.5}=1.74
$$

for that type of service. This means that the number of connections can be increased by $74 \%$ in comparison with those ones for $\eta_{1, U L}=0.5$ and a specific type of service. Note that, the mobile station range is reduced by $300 \mathrm{~m}$ for that service. So, if the coverage may be reduced in a cell, the capacity can be greater.

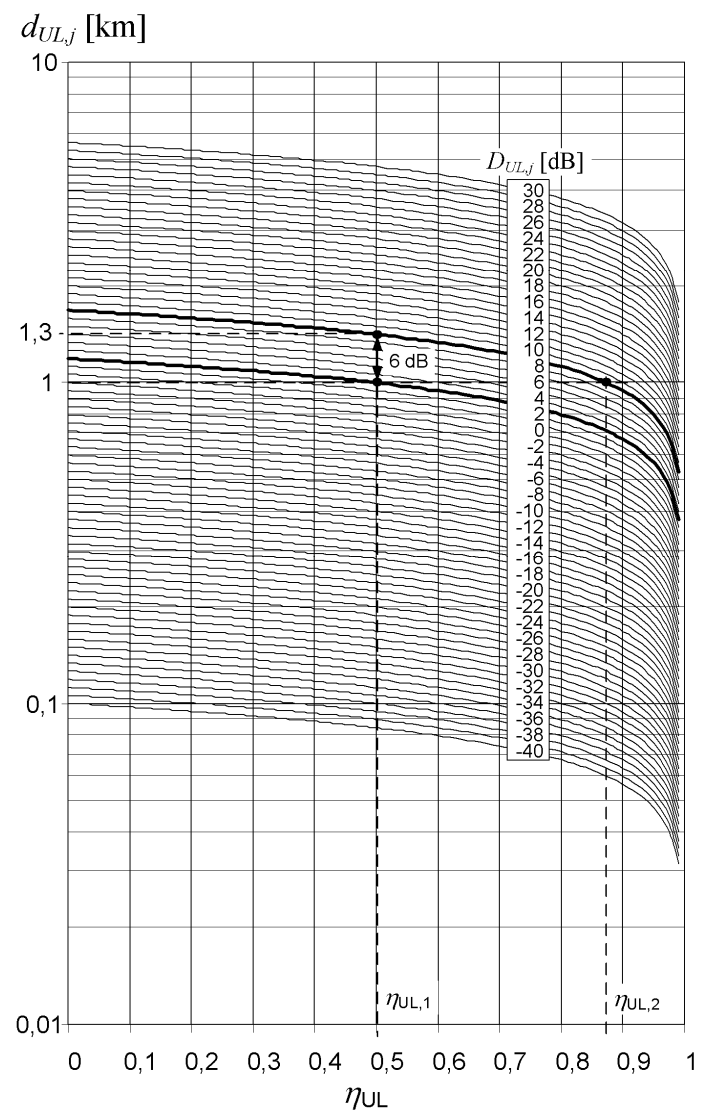

Fig. 4. Family of normalized coverage characteristics for uplink in OIP environment 
As we can see in Fig. 4, normalized coverage characteristics can be used to:

- e.g. if we would like to reduce the cell radius from $1.3 \mathrm{~km}$ to $1 \mathrm{~km}$, it can reduce the value of $D_{U L, j}$ by $6 \mathrm{~dB}$. If the network designer wants to do it, it can use lower antenna gain in the base station or it can decrease the mobile station transmitter power,

- result will be the same, if we not change the parameters of the radio interface, but allow an increase in load factor to 0.87 (instead of the previous 0.50 ).

Presented consideration shows, that the normalized coverage characteristics can be used to simple way of capacity-coverage trade-off estimation for the service implemented in $j$-th connection, for both the uplink and downlink. In addition, by using the characteristics (5) the capacity-coverage trade-off can be estimated for mixed services of any type in downlink.

Let's consider another example. Suppose that calculated for downlink the value of the parameter $D_{D L}=23 \mathrm{~dB}$. This corresponds to the bold characteristic in Fig. 5, passing through points " 1 " and " 2 ". At the point " 1 " of the characteristic, the base station

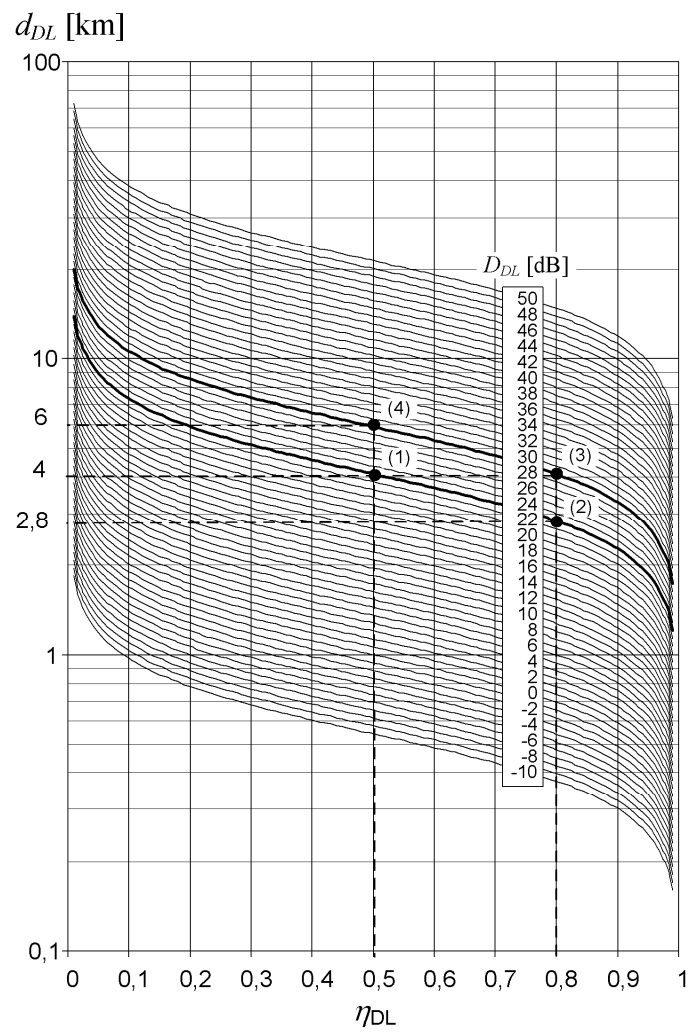

Fig. 5. Family of normalized coverage characteristics for the services of any type implemented in downlink (OIP environment) 
range is approximately $4 \mathrm{~km}$, with the assumed load equal to 0.5 . As we can see, by increasing the allowable load to about 0.8 coverage achieved will decrease to approximately $2.8 \mathrm{~km}$ (point " 2 "). If this value of the load is acceptable for a designer, it can allow the maximum load of 0.8 .

From the other side, if we would like to provide coverage at the same level as at the beginning of the analysis, we can proceed as follows. Increasing the values for $D_{D L}$ to $29 \mathrm{~dB}$ will allow us to obtain a range of approximately $4 \mathrm{~km}$, with an assumed load of 0.8 (point " 3 "), as we can see in Fig. 5. It can be achieved e.g. by increasing the base station transmitter power of about $6 \mathrm{~dB}$ or use the antenna with higher gain. We can therefore make the appropriate changes to the base station. Of course, with such changes is increasing coverage (for the load of 0.5 ), which in this case would be about $5.9 \mathrm{~km}$ (point "4"). Note, that a similar analysis also for the uplink must be done.

We can see that by measuring the load we are able to control capacity and coverage in various operational conditions of the radio interface. Using this method in the planning and operation of the network can simplify the radio resource management. Also, we can increase the efficiency of the use radio resources. The presented method of analysis can be performed for different types of services implemented in any propagation environment. Moreover, described method can be used to reconfigure the network, which may allow a temporary increase in capacity [3].

\section{Applications of the Load and Coverage Characteristics}

For design the $3 \mathrm{G}$ radio network both the load and coverage characteristics can be used. They may be applied for the verification of propagation and coverage conditions, both at network startup, as well as during its operation. By using the characteristics we can rapidly assess the necessary changes in the link budget parameters and thus, increase the efficiency of the use of radio resources. The presented method can be used for the simulation and measurement investigations.

It should be noted, that the use of load characteristics may significantly speed up the process of network design, because the consequences of changes in the value placed on the radio link parameters are directly visible. In addition, clearly visible is the effect of changes of these radio link parameters on another characteristics of the network: e.g. load, capacity, coverage etc.

Among the many applications of universal load characteristics we can mention:

- analysis of the allowable pathloss value for any configuration of link budget parameters,

- estimation of pathloss variation depending on the variability of the load,

- calculation of allowable pathloss for different values of the load and miscellaneous services of any type,

- determination of the extent of transmitter power variation in different operating conditions, for both the base stations and mobile stations.

Note that, the normalized coverage characteristics in any propagation environment and for any propagation loss model can be used. Furthermore, all of the listed applications of universal load characteristics can be used as an application of normalized coverage characteristics. 
Among the applications of normalized coverage characteristics we can mention in particular:

- analysis of the capacity-coverage trade-off for a given set of implemented services,

- calculation of coverage in different operating conditions, for various values of the load and services of any type,

- defining the coverage changes resulting from the modification of link budget parameters.

Of course, the primary purpose of the use of coverage characteristics is to designate the coverage, for both the base stations and mobile stations. In spite of the significant complexity of the WCDMA/FDD radio interface, the designation of capacitycoverage trade-off is very simple, when presented method is used.

\section{Summary}

The method of the estimation of capacity-coverage tradeoff presented in this paper is useful for both the planning and maintenance of the UMTS network. As seen, we can use the phenomenon of the trade-off to determine the necessary changes in the value of radio link parameters, depending on the volatility of the operating conditions in a radio network.

In the network planning process we can shape the cell coverage at the cost of capacity for the service of any type and for arbitrary propagation environment in order to utilize the radio resources in the most efficient way.

In the maintenance process we can easily evaluate the coverage when the traffic is increasing in some periods of time and borrow the resources (capacity) needed from neighbouring cells. Ultimately, if the traffic is increased permanently we can redesign the local network appropriately.

The presented method can be used for simple calculations, when the values of link budget parameters are modified in a real network. Also, to simulate the UMTS network in the expert systems it can be used.

\section{References}

1. Laiho-Steffens, J., Wacker, A., Aikio, P.: The Impact of the Radio Network Planning and Site Configuration on the WCDMA Network Capacity and Quality of Service. In: Proc. of IEEE Vehicular Technology Conference, VTC 2000 Fall (2000)

2. Laiho, J., Wacker, A., Novosad, T. (eds.): Radio Network Planning and Optimisation for UMTS, 2nd edn. Wiley \& Sons, Chichester (2006)

3. Gajewski, S.: Performance Analysis of the WCDMA /FDD Technique in the UMTS Terrestrial Radio Access Network, doctoral dissertation (in Polish), Gdansk University of Technology, Gdansk, Poland (2004)

4. Veeravalli, V., Sendonaris, A.: The Coverage - Capacity Tradeoff in Cellular CDMA Systems. IEEE Transactions on Vehicular Technology 48(5) (September 2002)

5. Gajewska, M., Gajewski, S.: Simulation Results of Dynamic Capacity Reallocation in Hierarchical Cell Structure of UMTS Network. Polish Journal on Environmental Studies 16(4B) (2007) 
6. Gajewski, S., Gajewska, M.: Downlink Capacity-Coverage Trade-off Estimation Based on Measurement of WCDMA/FDD Interface Load. Polish Journal of Environmental Studies 16(4B) (2007)

7. Shikh-Bahaei, M.R., Aghvami, A.H.: Joint Planning of Soft-capacity and Soft-coverage for 3G WCDMA Systems, 3G Mobile Communication Technologies, Conference Publication No. 477, IEE 2001 (2001)

8. ITU-R, Guidelines for Evaluation of Radio Transmission Technologies for IMT-2000, Recommendation ITU-R M.1225 (1997) 